\title{
Evaluation of perfusion by near-infrared fluorescence imaging in late pedicle obstruction of a parascapular flap to the lower extremity: A case report
}

\author{
Felix F. Strübing $M^{1} D^{1}$ Amir K. Bigdeli MD ${ }^{1}$ @ | Volker J. Schmidt $M^{1}$ | \\ Spyridoula Maraka $\mathrm{MD}^{2}$ | Ulrich Kneser $\mathrm{MD}^{1}$ | Dimitra Kotsougiani MD ${ }^{1} \odot$
}

${ }^{1}$ Department of Hand, Plastic and Reconstructive Surgery, Burn Center, BG Trauma Center Ludwigshafen, Hand and Plastic Surgery, University of Heidelberg, Heidelberg, Germany

${ }^{2}$ Division of Endocrinology and Metabolism, Center for Osteoporosis and Metabolic Bone Diseases, University of Arkansas for Medical Sciences and the Central Arkansas Veterans Health Care System, Little Rock, Arkansas

\section{Correspondence}

Dimitra Kotsougiani, Department of Hand, Plastic, and Reconstructive Surgery, BG

Trauma Center Ludwigshafen, Hand and Plastic Surgery, University of Heidelberg, Ludwig Guttmann Str., 1367071

Ludwigshafen, Germany.

Email: dkotsougiani@googlemail.com

\begin{abstract}
Late free flap failures due to pedicle obstruction are rare adverse events. The purpose of this report is to present a case in which the near-infrared indocyanine green video angiography (ICG-NIR-VA) was used to evaluate the perfusion in a compromised fasciocutaneous flap in the late postoperative period. A 55-year-old male patient, who initially suffered from an open tibial fracture, developed concurrent osteomyelitis with fistulae of the proximal tibia requiring excision. Reconstruction was achieved using a parascapular flap of $25 \times 8 \mathrm{~cm}$, anastomosed to an arterio-venous (AV) Loop in the adductor canal. On the 2nd postoperative day the patient developed a thrombosis of the AV-Loop. After thrombectomy, the postoperative course was uneventful. Seven weeks postoperatively the patient presented with a mottled, partially bluish and cold flap. Anticoagulation prophylaxis was stopped 2 days earlier. Angiography confirmed a thrombus in the arterial pedicle leading to flap ischemia. Near infrared guided fluorescence imaging revealed a delayed recapillarization of the proximal part of the flap closest to the vascular pedicle, albeit flap overall perfusion remained intact. Thus, no surgical intervention was undertaken. However, anticoagulation and prostaglandin therapy was initiated and the flap was salvaged. Genetic analysis uncovered a polymorphism in the prothrombin genes. The flap remained viable and without further complications until the last follow-up visit at 36 weeks after microsurgical reconstruction. The patient was fully mobilized with complete return to function. Near-infrared guided fluorescence imaging may be a viable tool for the assessment of late fasciocutaneous free flap complications and guide the decision-making process.
\end{abstract}

\section{1 | INTRODUCTION}

Free tissue transfer has become a routine procedure for reconstruction of complex lower extremity soft-tissue defects with failure rates below $5 \%$ (Xiong et al., 2016). It is believed that fasciocutaneous flap autonomization of the vascular pedicle is completed $\sim 4$ weeks after surgery and that secondary refinement procedures can be performed safely in the third month postoperatively(Kotsougiani et al., 2017).

Late free flap failure, occurring after the 7 th postoperative day, is a rare event. Only about $1 \%-2 \%$ of free flap compromises occur in the late postoperative period(Salgado et al., 2002; Wax \& Rosenthal, 2007). Many reasons have been reported, including thrombosis, infection and compression of the pedicle(Wax \& Rosenthal, 2007).

Frequently, the patients have been discharged before the onset of the first symptoms of late pedicle obstruction, impeding diagnostic measures. Furthermore, free flap salvage might be technically challenging in the late postoperative period, in particular in patients presenting belatedly. Therefore, some centers advise the patients to stay close to the area of the hospital for a certain period of time after microvascular free flap reconstruction(Largo et al., 2018). Conventional methods of diagnosing late free flap failure include clinical examination, computed tomographic angiography, conventional angiography and Doppler sonography(Granzow, Li, Caton, \& Boyd, 2015; Salgado, Moran, \& Mardini, 2009). Lately near-infrared indocyanine green video angiography (ICG-NIR-VA) has been used to evaluate free-flap perfusion(Holm et al., 2002). Intraoperatively it may detect arterial spasms, venous congestion or regional hypoperfusion of free flaps(Holm et al., 2002).

The purpose of this report is to present a case in which the ICGNIR-VA was used to evaluate the perfusion in a compromised fasciocutaneous flap in the late postoperative period. 

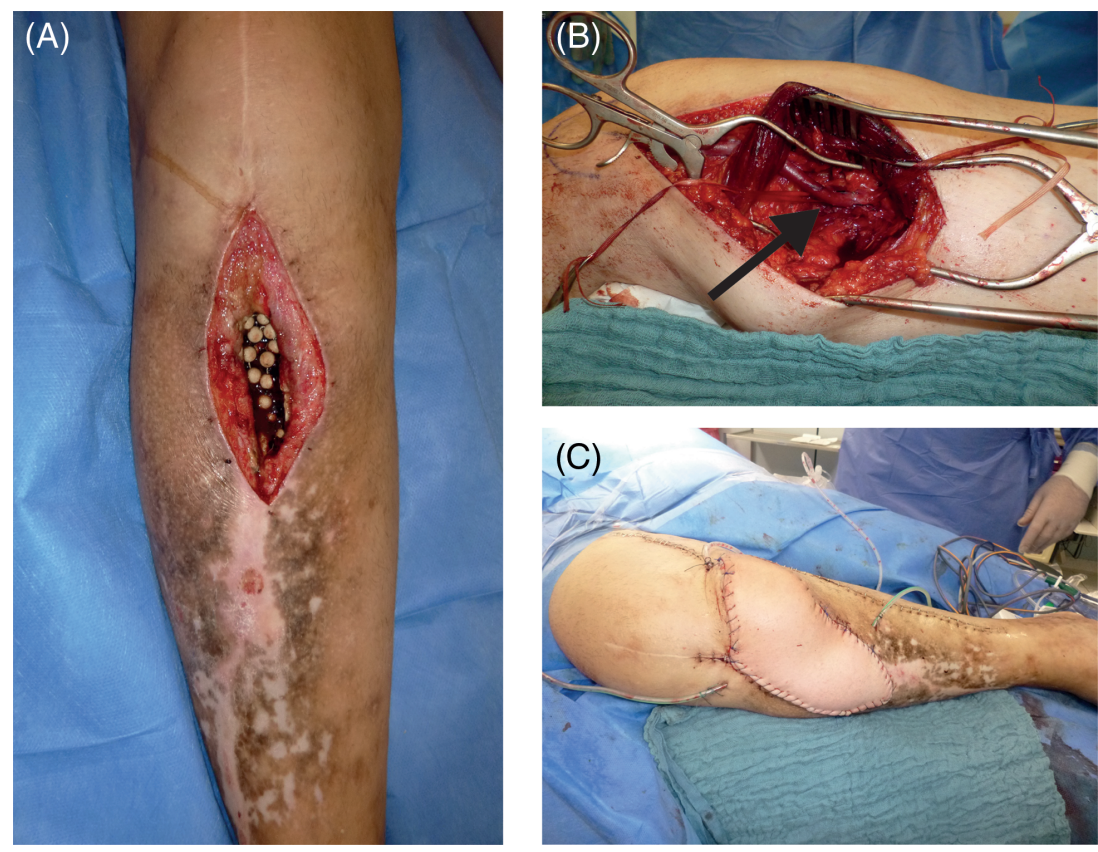

FIGURE 1 Preoperative and intraoperative aspect of the lower extremity defect, arterio-venous (AV)-loop and parascapular (PSC) flap: A, preoperative aspect of the wound on the proximal lower right leg, gentamicin-PMMA-chains were clearly visible in the opened tibial shaft; B, intraoperative aspect of the AV-loop in the adductor canal, the loop was well perfused; and C, the parascapular flap immediately after transfer and inlay of multiple drains

\section{2 | CASE REPORT}

A 55-year-old male patient with a history of smoking fell from a ladder and sustained a tibia fracture 15 years ago. Concurrent wound infections and chronic tibial osteomyelitis resulted in a tissue defect in the area of the knee and proximal third of the right tibia (Figure 1A). Microbiological analysis of bone samples showed Staphylococcus aureus susceptible to, among others, flucloxacillin. Therefore, the patient was put on flucloxacillin therapy for 6 weeks after radical debridement and insertion of Gentamicin-PMMA-chains. To achieve wound coverage, a free fasciocutaneous parascapular (PSC) flap of $25 \times 8 \mathrm{~cm}$ was microsurgically transplanted (Figure $1 \mathrm{C}$ ). Arterial and venous anastomoses were performed end-to-end to an arterio-venous (AV) loop (Figure 1B). For creation of the AV-loop, the great saphenous vein of the contralateral leg was harvested and anastomosed to the superficial femoral artery and the accompanying vein in the adductor canal. A bolus dose of 3,000 units of unfractioned heparin was administered intraoperatively for thrombosis prophylaxis. Since rotational thromboelastometry analysis was normal, low-molecular-weight heparin (LMWH) was used for postoperative deep vein thrombosis prophylaxis.

On the first postoperative day, the flap exhibited clinical signs of arterial insufficiency. Immediate reoperation verified a $10 \mathrm{~cm}$ long thrombus within the arterial part of the AV-loop, requiring subsequent thrombectomy. No technical problems, such as kinking of the pedicle, leakage, or compression of the arterial anastomosis were evident. The salvage procedure was successful and the further postoperative course was uneventful. Anticoagulation was consecutively continued using continuous intravenous unfractioned heparin infusions, aiming for a partial thromboplastin time of 40-45 s. Five days after revision, prophylactic anticoagulation was continued with $\mathrm{LMWH}(0.4 \mathrm{~mL}$ once daily). The patient was discharged from the hospital on the 15 th postoperative day.
On the 49th postoperative day, the patient noted that the flap had become partially bluish and cold. In the next morning, the patient presented at our outpatient clinic. No trauma was reported and the patient was in a healthy and good condition. The patient was wearing his custom-made compression garments for the lower extremity. According to the patient, LMWH prophylaxis was stopped 2 days earlier, corresponding to the 6th postoperative week. The clinical examination showed a partially bluish, cold, mottled flap in the proximal part of the parascapular flap, corresponding to the area of the medial knee, and raising the suspicion of a vascular flap pedicle thrombosis (Figure $2 \mathrm{~A}$ ). Computed tomographic angiography confirmed the suspected diagnosis, revealing a large thrombus with almost complete occlusion of the arterial side of the AV-loop starting $3 \mathrm{~cm}$ beyond the origin of the saphenous vein graft from the arteria femoralis superficialis. To assess the flap perfusion an ICG-NIR-VA system (Fluobeam 800, Fluoptics, Paris, France) was used that features a unique fusion image with the ability to overlay infrared images onto white light images with a colorcoded perfusion scale(Bigdeli et al., 2015). This allows the surgeon to analyze free flap perfusion on real-time and to assess hypoperfused flap areas(Bigdeli et al., 2015). Therefore, an area with known good perfusion, such as the untouched skin around the flap, was compared to the fluorescence signal strength of the flap. A relative fluorescence intensity of less than $33 \%$ was correlated to poor flap perfusion(Moyer \& Losken, 2012). Interestingly, all flap zones were sufficiently perfused, whereby the zone with the closest proximity to the flap pedicle-in the area of the medial knee-showed a delayed, albeit sufficient perfusion (Figure 3A). In consideration of the ICG-NIR-VA result we decided against surgical revision. Instead the patient was admitted to our hospital and put on therapeutic anticoagulation therapy for 7 days with LMWH (0.6 mL twice daily). In addition, Prostaglandin E1 (PGE1) was administered intravenously twice daily in a dose of $40 \mu \mathrm{g}$. To determine 

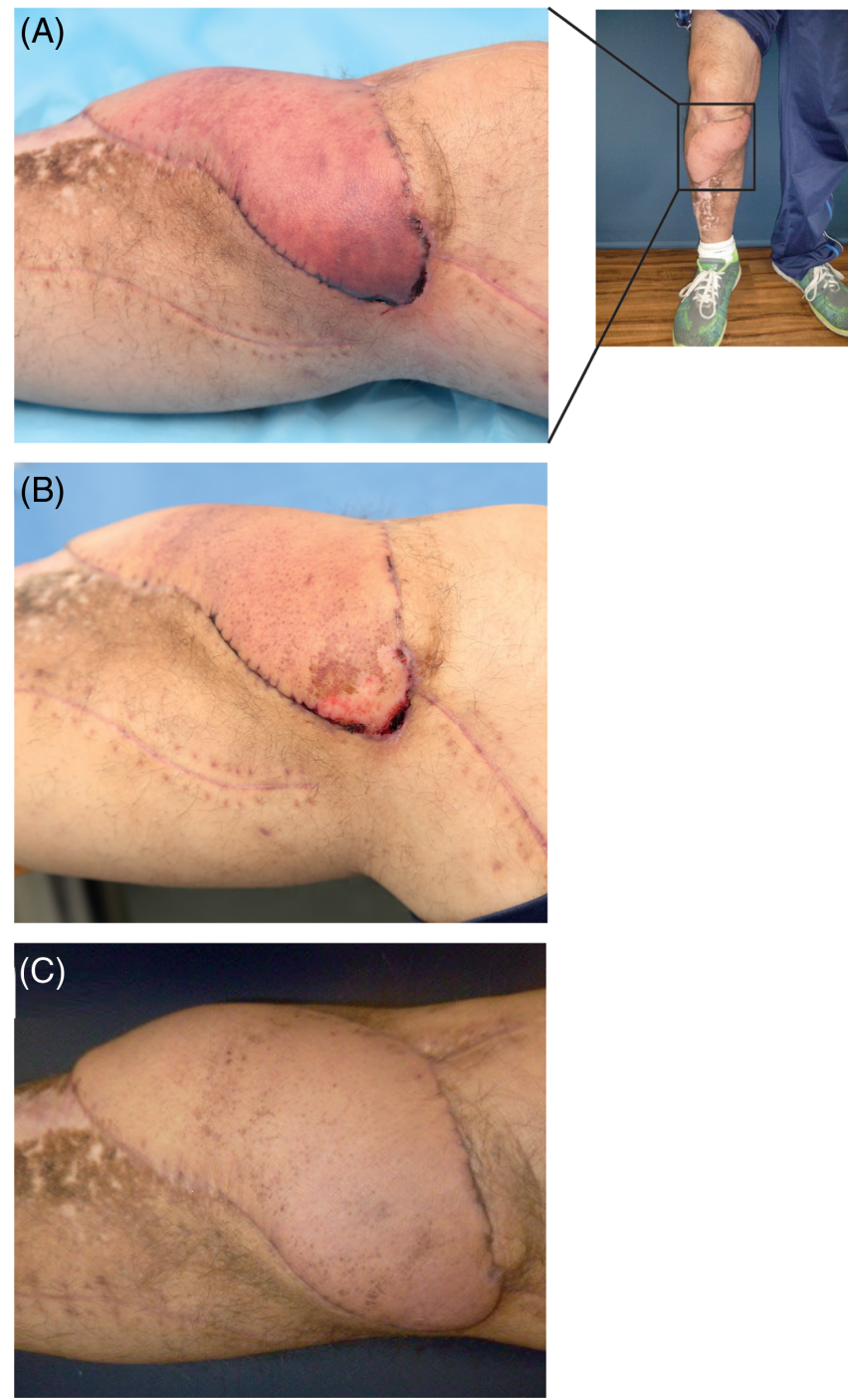

FIGURE 2 Clinical aspect of the parascapular (PSC) flap on the right lower leg of the patient: A, on hospital admission in the 7th week postoperative after PSC flap reconstruction, the flap was bluish and mottled in the proximal part; $\mathrm{B}$, on the day of hospital discharge (9th week postoperative) the bluish color had disappeared with remaining signs of epitheliolysis; and C, in the follow up examination 11 weeks after the reconstructive procedure, the soft tissue had recovered completely and all zones of the flap were viable

the reason of the recurring thrombotic events laboratory tests and genetic analysis for coagulopathies were performed. Elevated levels of factor VII, fibrinogen, and anti-phospholipid antibodies were found. Furthermore, it was revealed that the patient suffers from polymorphisms in the genes coding for prothrombin (Prothrombin G20210A wild type, Prothrombin A19911G heterozygous, Factor V A6755G heterozygous). The patient was discharged in the 9th week after microsurgical reconstruction, when the bluish color had disappeared with only remaining signs of epitheliolysis (Figure 2B).

Despite abrupt occlusion of the arterial axis of the AV loop, 1 month later, corresponding to 11 weeks after the microsurgical procedure, the entire flap was successfully integrated to the recipient site and showed an adequate perfusion pattern. The follow-up ICG-NIR$\mathrm{VA}$ at this time point verified a homogenous perfusion of the complete parascapular flap (Figures $2 \mathrm{C}$ and $3 \mathrm{~B}$ ). In the latest follow-up
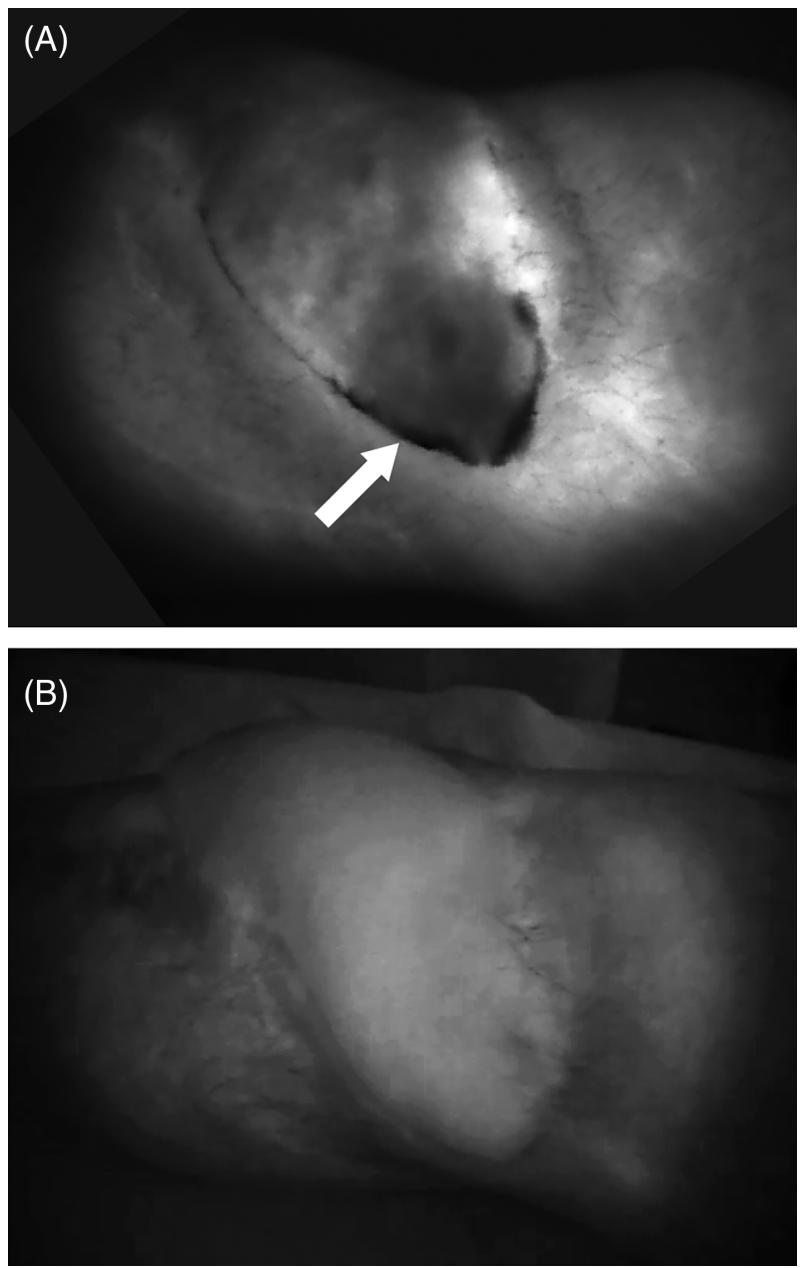

FIGURE 3 Near-infrared imaging of the parascapular (PSC) flap immediately following indocyanin green injection: $A$, on the day of hospital admission, 7 weeks after the reconstructive procedure the proximal part of the PSC flap showed signs of hypoperfusion (white arrow) and B, in the follow up examination 4 weeks after the intervention (11 weeks after the microsurgical procedure) the flap was well perfused in all zones

visit in the 36th week after the transplantation the PSC flap perfusion was intact. The patient was fully mobilized without the need for crutches and showed complete return to function. The patient had come back to his previous occupation as a forklift operator and was satisfied with the reconstructive outcome.

\section{3 | DISCUSSION}

Late free flap ischemia, occurring later than 1 week after the microsurgical reconstructive procedure, is a rare event(Wax \& Rosenthal, 2007). Reported causes are thrombosis of the anastomotic site, compression or injury of the pedicle, infection, and locoregional tumor recurrence.

The critical interval for free flap autonomization of the vascular pedicle differs between fasciocutaneous and muscle flaps. Experimental research in rats has shown a reliable fasciocutaneous autonomization as early as 5-10 days following reconstruction, whereby muscle flaps autonomy is reliable at 21 days(Bradshaw \& Wagels, 2017; Mücke et al., 2011). Accordingly, Yoon and Jones demonstrated in a 
systemic analysis an earlier flap survival of fasciocutaneous flaps when compared to muscle flaps after free flap pedicle obstruction(Yoon \& Jones, 2015). However, 4 weeks following surgery, autonomization of fasciocutaneous free flaps seems to be completed due to neovascularization and angiogenesis allowing secondary refinement procedures(Yoon \& Jones, 2015). In our case, on the contrary, ischemia of the parascapular flap was seen at over 7 weeks post-surgery. In particular, the flap area around the knee with the closest proximity to the vascular pedicle, exhibited clinical signs of hypoperfusion. A possible explanation might represent the ischemic gradient model, which describes an enhanced neovascularization in the area furthest from the pedicle(Murohara, 2003). Another hypothesis is that the traumatic and infectious background of recipient site might impair the process of neoangiogenesis, since the quality of the wound bed and flap randomization are associated(Salgado et al., 2002).

Salvage procedures of free flaps with a comprised perfusion pattern remain a great challenge with success rates ranging from $30 \%$ to $60 \%$ (Bui et al., 2007). The main therapeutic alternatives are take-back surgery with thrombectomy and conventional thrombolytic measures or minimal-invasive thrombolysis. In the latter, catheter-directed endovascular urokinase infusion is used to dissolve the thrombus(Trussler, Watson, \& Crisera, 2008). In addition, flap salvage might be achieved by conservative treatment as in our case(Yoon $\&$ Jones, 2015). Since the patient presented in our outpatient clinic almost $12 \mathrm{~h}$ after the first signs of flap ischemia, flap recovery from this situation is usually unlikely(Hanasono \& Butler, 2008). However, catheter directed thrombolysis using urokinase has shown to be successful in the late salvage of head and neck, breast, and extremity reconstruction and may have been considered as an therapeutic option in our case, if we would have not been satisfied with the ICGNIR-VA result(Parry et al., 2002; Trussler et al., 2008). Instead, we used a combination therapy, consisting of LMWH and PGE1 for flap salvage. Prostaglandin E has multiple effects on microcirculation with relevant anti-ischemic, fibrinolytic, and anti-inflammatary properties. Experimental work in rats has demonstrated the value of PGE1 for improvement of microcirculation in random pattern flaps(Tønseth Sneistrup, \& Berg, 2017). Furthermore, Hong et al. analyzed the effect of PGE1 on ischemia-reperfusion injury in musculocutaneous flaps in rats. Histological and immunohistochemical studies showed PGE1 to reduce leukocyte adhesion and to decrease expression of ICAM-1 on the surface of the endothelium after reperfusion, demonstrating a tissue protective effect of PGE1(Hong, Chung, \& Chung, 2001). Furthermore, in a clinical study Rodriguez Vegas et al. presented their antithrombotic pharmacologic treatment in free flap surgery which includes the intraoperative and postoperative administration of PGE1 (Vegas, Alonso, \& Saavedra, 2007). Faced with 2 consecutive thrombotic events in the postoperative course, we performed extensive laboratory and genetic analysis to reveal a coagulopathy disorder. The laboratory tests performed are similar to that of other institutions(Herrera et al., 2012). However, early coagulation diagnostics may have prevented the second thrombotic event and should be therefore reconsidered after early AV-loop thrombosis. Long-term anticoagulation therapy could be in these cases indicated. Since late pedicle obstruction can lead to free flap ischemia even months after surgery, patients should be informed about the clinical signs of late free flap ischemia during the discharge interview and counseled to present immediately in the case of such symptoms.

As early as 2002, Holm and colleagues published a prospective, clinical study of the use of near-infrared fluorescence imaging for intraoperative free flap evaluation(Holm et al., 2002). They were able to detect arterial spasms, venous congestion, or regional flap hypoperfusion. Recently, indocyanine green-based angiography has been introduced for skin-flap perfusion evaluation after mastectomy(Griffiths, Chae, \& Rozen, 2016). Furthermore, Mücke et al. presented the additional value of indocyanine green assisted videography in the early postoperative monitoring of transplanted epigastric flaps in an experimental study with rats with a positive predictive value of flap necrosis of $97 \%$ (Mücke et al., 2016). However, infusion of indocyanine green dye is not without risks and has some limitations. Possible adverse side effects are rare, but include headache, urticaria, hypotension, and cardiac arrest(Benya, Quintana, \& Brundage, 1989). Since many indocyanine green dyes contain sodium iodide, preoperative screening for hyperthyroidism is advised.

ICG-NIR-VA may represent a viable tool to guide the decisionmaking process in late fasciocutaneous free flap complications and needs further investigation to prove its reliability.

\section{ETHICAL APPROVAL STATEMENT}

This is a report of a clinical case with all investigations and treatment carried out to a high ethical standard. The case report includes routine, retrospective and anonymized patient's data, procedural parameters and outcomes and thus is in accordance with the Declaration of Helsinki without need for further ethical consultation.

\section{ORCID}

Amir K. Bigdeli (D) https://orcid.org/0000-0001-8852-5132 Dimitra Kotsougiani (D) https://orcid.org/0000-0003-2685-1105

\section{REFERENCES}

Benya, R., Quintana, J., \& Brundage, B. (1989). Adverse reactions to indocyanine green: A case report and a review of the literature. Catheterization and Cardiovascular Diagnosis, 17(4), 231-233.

Bigdeli, A. K., Gazyakan, E., Schmidt, V. J., Hernekamp, F. J., Harhaus, L., Henzler, T., ... Hirche, C. (2015). Indocyanine green fluorescence for free-flap perfusion imaging revisited: Advanced decision making by virtual perfusion reality in Visionsense fusion imaging angiography. Surgical Innovation, 23(3), 249-260. https://doi.org/10.1177/1553350615 610651

Bradshaw, K., \& Wagels, M. (2017). Perfusion of muscle flaps independent of the anatomical vascular pedicle: Pedicle autonomy. Journal of Plastic, Reconstructive \& Aesthetic Surgery, 70(11), 1547-1555. https://doi. org/10.1016/j.bjps.2017.05.049

Bui, D. T., Cordeiro, P. G., Hu, Q.-Y., Disa, J. J., Pusic, A., \& Mehrara, B. J. (2007). Free flap Reexploration: Indications, treatment, and outcomes in 1193 free flaps. Plastic and Reconstructive Surgery., 119(7), 2092-2100. https://doi.org/10.1097/01.prs.0000260598.24376.e1

Granzow, J., Li, A. I., Caton, A., \& Boyd, J. B. (2015). Free flap survival following failure of the vascular pedicle. Annals of Plastic Surgery, 75(1), 44-48. https://doi.org/10.1097/SAP.0000000000000136

Griffiths, M., Chae, M. P., \& Rozen, W. M. (2016). Indocyanine green-based fluorescent angiography in breast reconstruction. Gland Surgery, 5(2), 133-149. https://doi.org/10.3978/j.issn.2227-684X. 2016.02.01 
Hanasono, M. M., \& Butler, C. E. (2008). Prevention and treatment of thrombosis in microvascular surgery. Journal of Reconstructive Microsurgery, 24(05), 305-314. https://doi.org/10.1055/s-20081080530

Herrera, F. A., Lee, C. K., Kryger, G., Roostaeian, J., Safa, B., Lohman, R., ... Walton, R. (2012). Microsurgery in the hypercoagulable patient: Review of the literature. Journal of Reconstructive Microsurgery, 28(05), 305-312. https://doi.org/10.1055/s-0032-1311687

Holm, C., Tegeler, J., Mayr, M., Becker, A., Pfeiffer, U. J., \& Mühlbauer, W. (2002). Monitoring free flaps using laser-induced fluorescence of indocyanine green: A preliminary experience. Microsurgery, 22(7), 278-287. https://doi.org/10.1002/micr.10052

Hong, J. P., Chung, Y. K., \& Chung, S.-H. (2001). The effect of prostaglandin E1 versus ischemia-reperfusion injury of musculocutaneous flaps. Annals of Plastic Surgery, 47(3), 316-321.

Kotsougiani, D., Platte, J., Bigdeli, A. K., Hoener, B., Kremer, T., Kneser, U., \& Harhaus, L. (2017). Evaluation of 389 patients following free-flap lower extremity reconstruction with respect to secondary refinement procedures. Microsurgery, 79(Suppl 3), 371-250. https:// doi.org/10.1002/micr.30219

Largo, R. D., Selber, J. C., Garvey, P. B., Chang, E. I., Hanasono, M. M., Yu, P., ... Baumann, D. P. (2018). Outcome analysis of free flap salvage in outpatients presenting with microvascular compromise. Plastic and Reconstructive Surgery, 141(1), 20e-27e. https://doi.org/10.1097/PRS. 0000000000003917

Moyer, H. R., \& Losken, A. (2012). Predicting mastectomy skin flap necrosis with Indocyanine green angiography: The gray area defined. Plastic and Reconstructive Surgery., 129(5), 1043-1048. https://doi.org/10. 1097/PRS.0b013e31824a2b02

Mücke, T., Borgmann, A., Wagenpfeil, S., Günzinger, R., Nöbauer, C., Lange, R., ... Wolff, K. D. (2011). Autonomization of epigastric flaps in rats. Microsurgery, 31(6), 472-478. https://doi.org/10.1002/micr. 20892

Mücke, T., Fichter, A. M., Schmidt, L. H., Mitchell, D. A., Wolff, K.-D., \& Ritschl, L. M. (2016). Indocyanine green videoangiography-assisted prediction of flap necrosis in the rat epigastric flap using the flow ${ }^{\circledR} 800$ tool. Microsurgery, 37(3), 235-242. https://doi.org/10.1002/micr. 30072

Murohara, T. (2003). Angiogenesis and vasculogenesis for therapeutic neovascularization. Nagoya Journal of Medical Science, 66(1-2), 1-7.

Parry, D., Byrne, P., Scott, D. J. A., Kessel, D., Robertson, I., Patel, J., \& Batchelor, A. (2002). Pharmacological salvage of a combined distal bypass and free flap with catheter-directed thrombolysis. British
Journal of Plastic Surgery., 55(2), 140-144. https://doi.org/10.1054/ bjps.2002.3744

Salgado, C. J., Moran, S. L., \& Mardini, S. (2009). Flap monitoring and patient management. Plastic and Reconstructive Surgery, 124(6 Suppl), e295-e302. https://doi.org/10.1097/PRS.0b013e3181bcf07b

Salgado, C. J., Smith, A., Kim, S., Higgins, J., Behnam, A., Herrera, H. R., \& Serletti, J. M. (2002). Effects of late loss of arterial inflow on free flap survival. Journal of Reconstructive Microsurgery, 18(7), 579-584. https://doi.org/10.1055/s-2002-35095

Tønseth, K. A., Sneistrup, C., \& Berg, T. M. (2017). Prostaglandin E1 increases microcirculation in random pattern flaps on rats measured with laser Doppler perfusion imaging. Plast Reconstr Surg Glob Open., 5(1), e1202. https://doi.org/10.1097/GOX.0000000000001202

Trussler, A. P., Watson, J. P., \& Crisera, C. A. (2008). Late free-flap salvage with catheter-directed thrombolysis. Microsurgery, 28(4), 217-222. https://doi.org/10.1002/micr.20480

Vegas, J. M. R., Alonso, M. E. R., \& Saavedra, P. P. T. (2007). PGE-1 in replantation and free tissue transfer: Early preliminary experience. Microsurgery, 27(5), 395-397. https://doi.org/10.1002/micr.20377

Wax, M. K., \& Rosenthal, E. (2007). Etiology of late free flap failures occurring after hospital discharge. The Laryngoscope, 117(11), 1961-1963. https://doi.org/10.1097/MLG.0b013e31812e017a

Xiong, L., Gazyakan, E., Kremer, T., Hernekamp, F. J., Harhaus, L., Saint-Cyr, M., ... Hirche, C. (2016). Free flaps for reconstruction of soft tissue defects in lower extremity: A meta-analysis on microsurgical outcome and safety. Microsurgery, 36(6), 511-524. https://doi.org/10. 1002/micr.30020

Yoon, A. P., \& Jones, N. F. (2015). Critical time for neovascularization/angiogenesis to allow free flap survival after anastomotic thrombosis without surgical intervention. Plastic and Reconstructive Surgery., $136(4$ Suppl), 19. https://doi.org/10.1097/01.prs.0000472296.47202.d8

How to cite this article: Strübing FF, Bigdeli AK, Schmidt VJ, Maraka S, Kneser U, Kotsougiani D. Evaluation of perfusion by near-infrared fluorescence imaging in late pedicle obstruction of a parascapular flap to the lower extremity: A case report. Microsurgery. 2018;38:912-916. https://doi.org/10.1002/ micr.30366 